\title{
Placental abnormalities in congenital heart disease
}

\author{
Nickie N. Andescavage ${ }^{1,2}$, Catherine Limperopoulos ${ }^{2,3,4}$ \\ ${ }^{1}$ Division of Neonatology, Children's National Health System, Washington, DC, USA; ${ }^{2}$ Department of Pediatrics, George Washington University \\ School of Medicine, Washington, DC, USA; ${ }^{3}$ Division of Diagnostic Imaging \& Radiology, Children's National Health System, Washington, DC, \\ USA; ${ }^{4}$ Department of Radiology, George Washington University School of Medicine, Washington, DC, USA \\ Contributions: (I) Conception and design: C Limperopoulos; (II) Administrative support: C Limperopoulos; (III) Provision of study materials or \\ patients: None; (IV) Collection and assembly of data: All authors; (V) Data analysis and interpretation: All authors; (VI) Manuscript writing: All \\ authors; (VII) Final approval of manuscript: All authors. \\ Correspondence to: Catherine Limperopoulos, PhD. Children's National Health System, 111 Michigan Ave. NW, Washington, DC 20008, USA. \\ Email: climpero@childrensnational.org.
}

\begin{abstract}
Congenital heart disease (CHD) remains the most common birth defect in infants, and critical CHD is associated with significant rates of morbidity and mortality. With the advent of powerful yet noninvasive advanced fetal imaging, it is becoming increasingly evident that the presence of CHD in utero disrupts typical development and contributes to the lifelong morbidity in this population. Across healthy and high-risk populations, intrauterine influences can permanently alter fetal development that may manifest in complex morbidities later in life, the so-called fetal-onset-of-adult-disease (FOAD) phenomenon. The placenta plays a critical role in not only supporting fetal development, but also by adapting to specific intrauterine conditions. The role of placental health, adaptation and dysfunction, however, in CHD is not well understood. In this article, we will review current evidence relating placental health in CHD, appraise existing knowledge-gaps in the field and highlight promising new avenues to better understand the impact of placental function on fetal well-being. We will review evidence of ex vivo human placental studies that describe abnormal placental findings in pregnancies complicated by CHD, as well evidence for in vivo assessments of the human placenta. While overall clinical in vivo assessments of placental development are rather limited, we will also review emerging evidence from advanced quantitative and functional magnetic resonance imaging that are bringing new insights into placental structure and function throughout gestation. By providing novel information about placental development, we can now explore the maternal-fetalplacental connection in greater detail, and better understand the multi-factorial mechanisms that may contribute to adverse outcomes seen in survivors of CHD.
\end{abstract}

Keywords: Placenta; congenital heart disease (CHD); imaging; ultrasound; magnetic resonance imaging

Submitted Oct 20, 2020. Accepted for publication Nov 05, 2020.

doi: $10.21037 /$ tp-20-347

View this article at: http://dx.doi.org/10.21037/tp-20-347

\section{Introduction}

The placenta is a critical organ necessary for the healthy development of any fetus; not only does it provide the necessary nutrients to promote growth and clear the fetal circulation of metabolic byproducts, it serves as gatekeeper to protect the fetus from a hostile intrauterine environment-including toxic stress, infection, inflammation and other damaging mediators, and to regulate early metabolic and endocrine functions (1-8). Deviations from normal placental function, especially if coupled with adverse intrauterine exposures, can result in significant physiologic changes of fetal development that increase the risk of cardiovascular, metabolic and neuropsychiatric disease across the lifespan $(9,10)$. For children born with congenital heart disease (CHD), placental dysfunction is yet another mechanism by which 


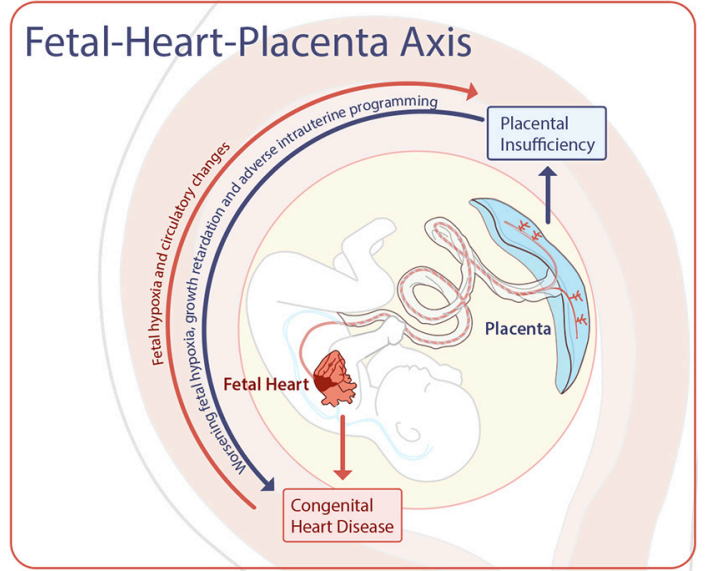

Figure 1 A diagram of the Fetal-Heart Placenta Axis, where fetal hypoxia and circulatory changes from congenital heart disease may contribute to placental insufficiency (red arrow), while ongoing placental insufficiency may further contribute to worsening fetal hypoxia, growth retardation and adverse intrauterine programming (blue arrow).

typical development may be disrupted, and a potential contributor to the lifelong morbidity and mortality seen in this fragile population.

The placenta-heart axis refers to the parallel development of the placenta and heart, two critical vascular structures with a shared ontogeny. Molecular and genetic studies have identified multiple shared pathways critical to both early placental and cardiac development, including critical micronutrients, such as folate $(11,12)$. Early changes in the embryonic development of the fetal and placental circulations can influence placental shape, size, cord insertion and ultimately placental efficiency (12). Similarly, spiral artery remodeling of the maternal uterine circulation also occurs within the first few weeks of pregnancy, and if this process goes awry increases the risk of pre-eclampsia, fetal growth restriction (FGR), preterm labor and stillbirth (12). In addition, there is potential for ongoing placental injury from poor implantation, hypoxia and circulatory changes that can result in "multi-hit" mechanisms of placental dysfunction and fetal harm (Figure 1). In the sections below, we will review the supporting clinical evidence of placental injury and mal-development in CHD, as well as promising new fields of investigation to better understand the clinical implications of placental health and dysfunction in the fetus with CHD.

\section{Ex vivo studies: placental pathology}

\section{Placental weight}

Placental pathology can provide great insight into intrauterine conditions, especially for high risk pregnancies, yet relatively little is known about placental pathology in CHD. Among the few studies reported, several of these have noted smaller placentas in newborns born with CHD (13-15). One early study of placental pathology in CHD from the Danish Medical Birth Registry compared placental findings in newborns with and without CHD (13). In this work, the authors found that mean placental weight was lower in newborns with CHD compared to newborns without CHD, with the greatest decrease in placental weight was in infants with tetralogy of Fallot (TOF), followed by double-outlet right ventricle (DORV) and major ventricular septal defects (VSD) (13). Another smaller study specific to hypoplastic left heart syndrome (HLHS) also found a significant reduction in placental weight in infants with HLHS compared to age matched controls (15). Similarly, Rychik et al. described placental characteristics among 4 sub-types of CHD and found that $77 \%$ of CHD cases had placental weights less than the $10^{\text {th }}$ centile for gestational age and nearly half had placental weights less than the $3^{\text {rd }}$ centile (14). Among healthy and high-risk populations, placental size is highly associated with fetal growth, with over a third of birth weight variation due to placental weight alone (16). Birth weight variation, in turn, is associated with neonatal outcomes, with increased risk of adverse outcomes for infants born small for gestational age (SGA) (17-19).

\section{Placental efficiency}

Two of these studies further evaluated the relationship between placental weight and birth weight specifically in CHD $(13,14)$. In the Danish Birth Registry study, the authors also described a significant association between placental weight and both birth weight and head circumference, and the case series on HLHS also noted both decreased birth weight and placental weight when compared to controls $(13,15)$. Interestingly, the association between placental weight and fetal growth varied significantly across sub-groups, with the greatest association among infants with TOF, DORV, major VSD and lowest among infants with transposition of the great arteries (TGA) or (HLHS) (13). Rychik et al. also presented the placental 
A

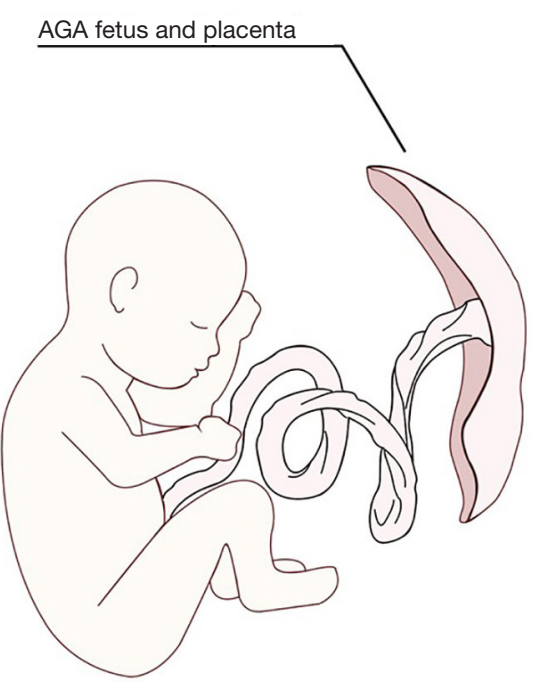

B

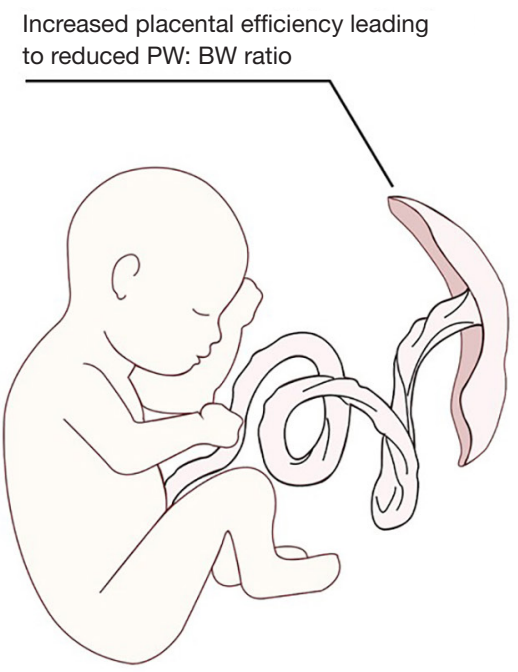

Figure 2 A diagram of placental efficiency. Panel (A) shows a typically developing fetus and placenta (appropriate for gestational age, AGA), while panel (B) shows a smaller placenta relative to fetal development, that would result in a lower placental weight (PW) to birth weight (BW) ratio. A reduced PW:BW ratio may reflect a highly efficient placenta that can support adequate fetal growth despite smaller placental size.

weight to birth weight ratio (PW:BW) (14). Typically, the $\mathrm{PW}: \mathrm{BW}$ has been cited as a marker of placental efficiency (Figure 2), with reduced ratios reflecting more efficient nutrient transfer from placental to fetus (9). In the study by Rychik et al., the PW:BW was lower in CHD compared to expected norms, particularly for infants with TGA (14). This is in contrast to the findings in a case-control study of infants with and without CHD by Albalawi et al., that found no different in the absolute $\mathrm{PW}: \mathrm{BW}$ ratio between infants with CHD and healthy controls (20). The study by Albalawi, however did note increased rates of fetal growth restriction among CHD cases, and for all subjects with FGR (cases and controls), the PW:BW ratio was higher in the setting of FGR (20). These conflicting findings may represent limitations from inherent differences in CHD, particularly when grouping distinct CHD diagnoses into sub-types for adequate power analysis. While the utility of the PW:BW as a surrogate of placental efficiency has been questioned $(9,21)$, it nonetheless has been linked with adverse cardiovascular health in adults (9). The relevance of these findings, however, in CHD, remains poorly understood.

\section{Placental shape}

Albalawi et al. also noted significant differences in cord insertion sites between CHD placentas and healthy controls (20), with higher rates of eccentric, marginal and velamentous insertion sites in CHD (20). Sub-group evaluation noted that the relative risk of CHD subtype in the setting of abnormal cord insertion was highest for conotruncal defects (relative risk, $\mathrm{RR}=3.08$ ), followed by left heart disease, namely HLHS and coarctation of the aorta $(\mathrm{RR}=2.4)$ and right heart disease, including Ebstein anomaly, tricuspid atresia, pulmonary atresia with intact ventricular septum, and critical pulmonary stenosis $(\mathrm{RR}=2.22)$ (20). Abnormal cord insertions sites, particularly marginal and velamentous insertion sites, have been associated with adverse pregnancy outcomes, including preterm birth, SGA infants, low birth weight (LBW) infants, emergency cesarean delivery and intrauterine fetal death, with velamentous cord insertion sites carrying the greatest risk $(22,23)$. Similarly, eccentric and peripheral cord insertion sites have been variably associated with SGA and LBW (24), premature birth and decreased placental weight z-score (Figure 3) (25).

\section{Histopathology}

Several placental abnormalities have been noted on pathologic examination of CHD placentas. In the study by Rychik et al., the most common lesion seen was thrombosis in $41 \%$ of CHD placentas as well as infarction (17\%) (14). On histopathology, higher rates of both maternal and fetal 
A

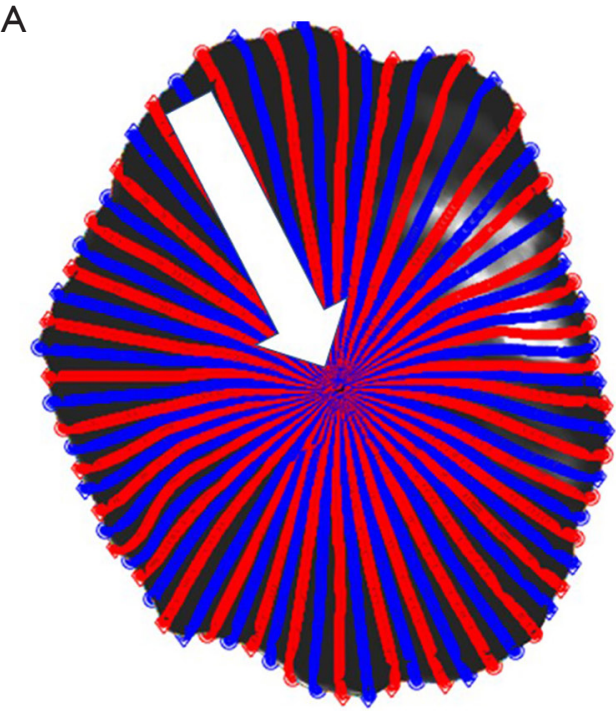

B

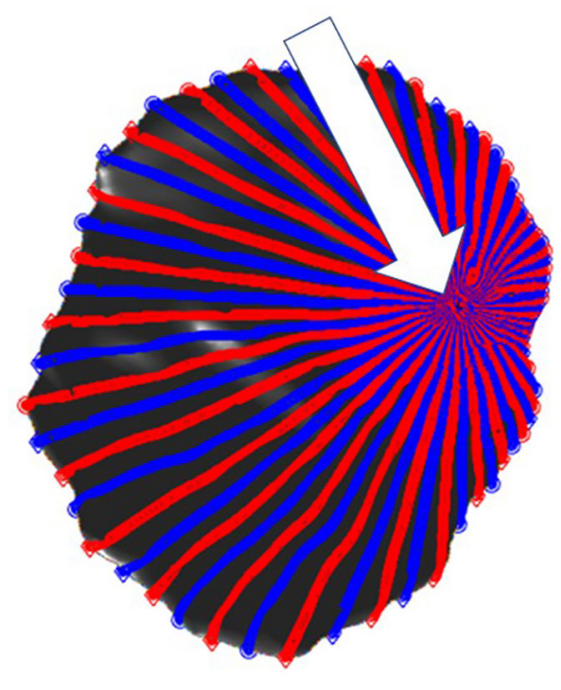

Figure 3 Three-dimensional reconstructions of in vivo placentas from MRI images, with the cord insertion site highlighted by a white arrow. Panel (A) demonstrates a placenta with central cord insertion site, and relatively symmetric fetal surface radii, while Panel (B) demonstrates a placenta with eccentric cord insertion site, and asymmetric fetal surface radii. These metrics have been used to develop a cord centricity score (unpublished data), as eccentric cord insertion sites are associated with growth disturbances of both the infant and placenta.

vascular malperfusion (MVM and FVM) were seen in a case control study of placentas from pregnancy terminations due to severe CHD compared to controls (26). FVM represents a group of placental lesions that can result from abnormal fetal perfusion to the villous parenchyma of the placenta. The most common cause of FVM is thought to be due to umbilical cord obstruction that limits fetal perfusion of the placenta, but CHD with accompanying flow abnormalities, cardiac insufficiency, and hyperviscosity from chronic hypoxia can also result in FVM (27). Segmental FVM consists of fetal vascular thrombi, villous stromalvascular karyorrhexis subsequent avascular and ischemic villi (27). Regardless of the etiology, the mechanisms behind common lesions of FVM are venous congestion and stasis that results in focal ischemia and thrombosis, and in particular, segmental FVM has been associated with several adverse outcomes, including FGR and intrauterine fetal demise (27). Rychik et al., also noted significant rates of chorangiosis (18\%) and villous immaturity (15\%) (14). Choriangiosis is characterized by increased vascularization of the distal villi, thought to occur after chronic hypoxia, and has been associated with avascular-ischemic villi seen in FVM (28) while villous immaturity is a lesion commonly seen in pregnancies complicated by diabetes mellitus, FGR, congenital malformations, and late gestational hypoxia (29). The Jones study of HLHS also noted increased fibrin and syncytial nuclear aggregates or syncytial knots in HLHS compared to controls (15). Both abnormalities of villous maturation and increased syncytial knots are associated with MVM. MVM, in contrast to FVM, is a constellation of placental findings that represent injury from altered uterine and intervillous flow from the maternal circulation. MVM is seen most often with preeclampsia and other hypertensive disorders of pregnancy, as well as spontaneous preterm birth and FGR. Interestingly, a study of 279 women carrying fetuses with CHD found significantly higher incidences of preeclampsia (OR 5.96), severe FGR (OR 3.32) and still birth (LR 9.45) compared to uncomplicated pregnancies (30). Collectively, these pathologic findings suggest that fetal flow anomalies and chronic hypoxia resulting in FVM, along with placental implantation anomalies resulting in MVM, may contribute to additional placental injury in CHD.

\section{In utero studies}

\section{Doppler sonography}

A common clinical approach to assess in vivo placental health is through Doppler interrogation of the umbilical arteries, either alone or relative to the fetal circulation as the cerebroplacental ratio (CPR) $(31,32)$. In certain types 
of placental pathology, blood flow through the placenta may encounter increased resistance and thus increasing the Doppler measures of the umbilical artery $(33,34)$. Changes in the CPR reflect redistribution of the fetoplacental circulation, and can reflect early adaptation to placental insufficiency, but also decreased Doppler measures of the middle cerebral artery (MCA) (32). Furthermore, abnormal CPR values have been associated with adverse perinatal and neurologic outcomes (31). In one study by Donofrio et al., only $44 \%$ of fetuses with CHD were found to have normal CPR values, with increased rates of abnormal MCA Dopplers (35). In this study, larger fetuses with CHD with a reduced CPR also had smaller head circumferences (35). This is in contrast to another study of pregnancies complicated by fetal CHD, serial measures of fetal biometry and cerebroplacental Doppler flow were measured in the second half of pregnancy showed normal Doppler flow patterns, and did not find any significant relationship between placental function (as measured by Doppler studies of the UA and CPR) and fetal head growth (36). More specifically, even though MCA Doppler values varied between sub-groups of CHD, there was no association between MCA Doppler values and head growth in this population of CHD (36). Differences between studies may reflect differences in sample size (44 vs. 181, respectively), but again, also differences in CHD sub-types and sub-group classification. Similarly, the study by Rychik et al., that described placental abnormalities in CHD on pathologic examination, did not find a significant association between fetal Doppler measures and postnatal placental anomalies (14). Despite the relatively few studies on Doppler sonography in CHD, there is no clear connection between fetal Doppler findings and placental health. The one study that did identify an association between increased rates of abnormal CPR values in CHD noted that UA values did not differ significantly between groups, with differences in MCA Dopplers driving differences in CPR (35). However, Doppler assessments of the UA remain indirect measures of placental health and may explain the variability in reliably predicting adverse pregnancy outcomes (32).

\section{Magnetic resonance imaging (MRI)}

The application of in vivo MRI in the living fetus has augmented prenatal sonography for the clinical diagnosis of many maternal-fetal conditions. Advances in both the application and interpretation of advanced MRI sequences are providing unparalleled access to detailed placental structural and functional development in healthy and high-risk pregnancies, including those complicated by fetal CHD.

\section{Placental volume}

In one study of in vivo placental imaging, 43 cases of fetal CHD were compared to 112 healthy controls (37). For all subjects, no placental anomalies were detected on conventional MRI and while overall placental volume did not differ significantly between groups, placental volume was positively associated with gestational age at birth, as well as birth weight in fetuses with CHD (37). Furthermore, the relationship between placental volume and birthweight, a proxy for placental efficiency in supporting fetal growth, revealed different trajectories between fetuses with CHD and healthy controls, suggesting functional differences in the CHD placenta (37). Interestingly, this study did not find an association between fetal brain volume and placental volume in CHD (37), although a comparable study of in vivo placental volumes and fetal brain volumes in healthy pregnancies and pregnancies complicated by fetal growth restriction found a positive association between placental volume and both total brain and cerebral volumes (38), further evidence to suggest that the normal associations between placental growth and fetal growth are disrupted in CHD.

\section{Placental perfusion}

Velocity-selective arterial spin labeling (VSASL) is a noninvasive technique to quantify global and regional perfusion (Figure 4); this technique has been successfully applied to the study of placental development in CHD (39). In this study, global placental perfusion decreased significantly over the second half of gestation in CHD, while regional variation in placental perfusion increased; most importantly these findings were not seen healthy controls (39). While the etiology of these differences are unclear, changes in placental perfusion in the second half of pregnancy may represent late manifestations of early developmental deviations or evidence of progressive placental dysfunction from abnormalities of the fetal circulation. These findings nonetheless support distinct functional aberrations of the CHD placenta, and emerge at a time of exponential fetal growth. Any changes in placental function that may accompany these changes in perfusion can increase the risk of late-onset fetal growth restriction, which increases 

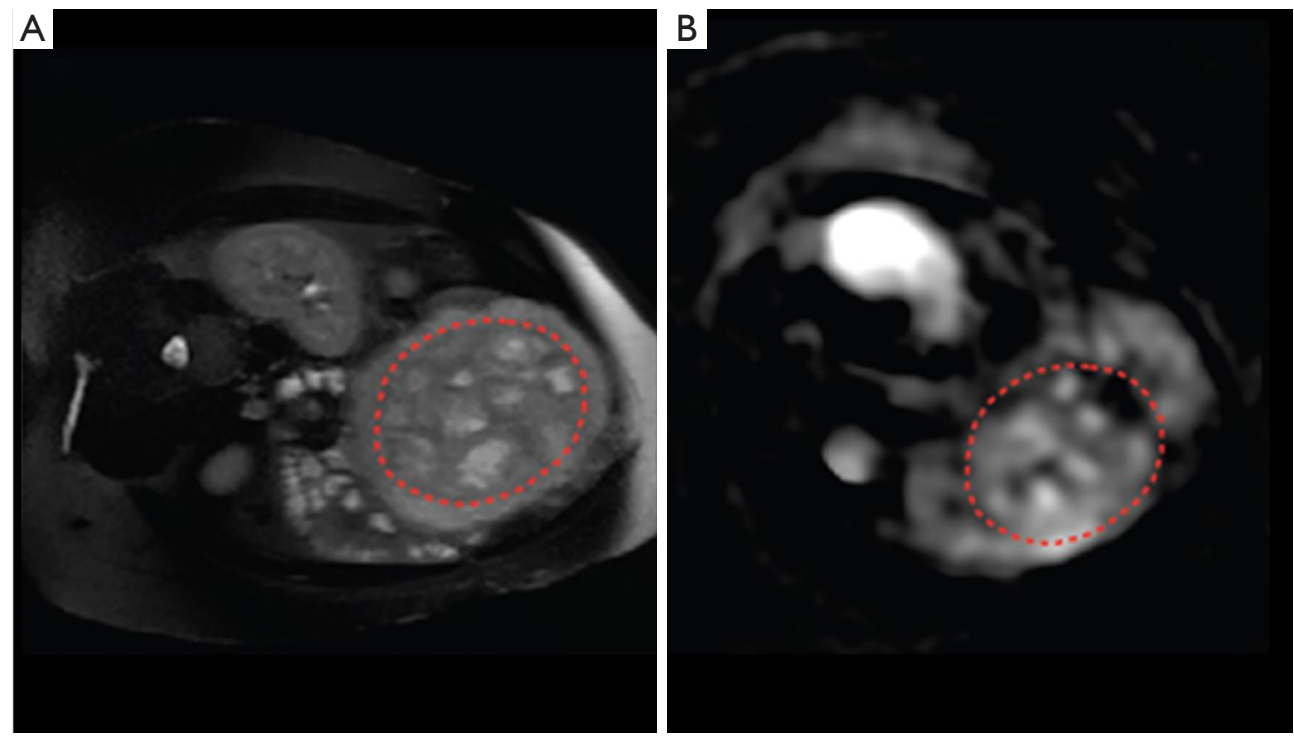

Figure 4 Anatomic (A) and arterial spin labeling images (ASL) (B) of the placenta in a pregnant woman with fetal congenital heart disease diagnosis, with the placenta outlined by the dotted line.

the risk of fetal distress, neonatal acidosis, and perinatal hypoxic injury, as well as long-term neurodevelopment and metabolic impairments (40).

\section{Feto-placental oxygenation}

Several MR sequences can estimate oxygen saturation and blood flow, all without the exogenous administration of contrast, including phase-contrast MRI, T2 mapping and blood-oxygen level dependent (BOLD) imaging. The successful application of these techniques to the fetus has allowed for the direct, yet non-invasive, interrogation of fetal-placental oxygenation. In a study by Sun et al., imaging flow and oximetry data were used to calculate umbilical and fetal vessel oxygen content, delivery and consumption in fetuses with CHD (41). This study revealed a significant decrease in oxygen content returning to the fetus from the placenta, which resulted in decreased cerebral oxygen delivery and decreased fetal brain volumes (41). Another study by You et al., used BOLD imaging during a maternal hyperoxia design (Figure 5) to measure regional oxygenation in healthy controls and pregnancies complicated by CHD (42). In this study, placental oxygenation increased significantly during maternal hyperoxia for all fetuses, and fetuses with single-ventricle CHD had the greatest response to maternal hyperoxia (42). This study suggests that the placenta has the capacity for increased oxygen delivery under certain circumstances, but also highlights the hypoxic state of the SV feto-placental unit at baseline.

While there are only a handful of advanced MRI studies on placental structure and function in CHD, collectively, these works provide hitherto unseen insights into in vivo placental structure and function and offer a promising opportunity to continue to understand the complex relationship between $\mathrm{CHD}$ and the placenta.

\section{Conclusions \& future directions}

It is becoming increasingly evident that placental structure and function is impaired in CHD, however much less is known regarding the onset and mechanism of placental injury and/or maldevelopment. Despite advances in fetal diagnostic techniques, there are no current clinical tools that directly and non-invasively assess placental function in utero (43). This remains a major void in the field given the significant role of placental health to fetal well-being. Filling this void is necessary to enhance our ability to optimize fetal growth and long-term health outcomes, including in infants with CHD. Emerging tools intended to detect early deviations of normal placental function, such as those discussed in this review, may provide new insights into placental health overall, but especially to pregnancies complicated by CHD.

Future research intended to understand the timing, 

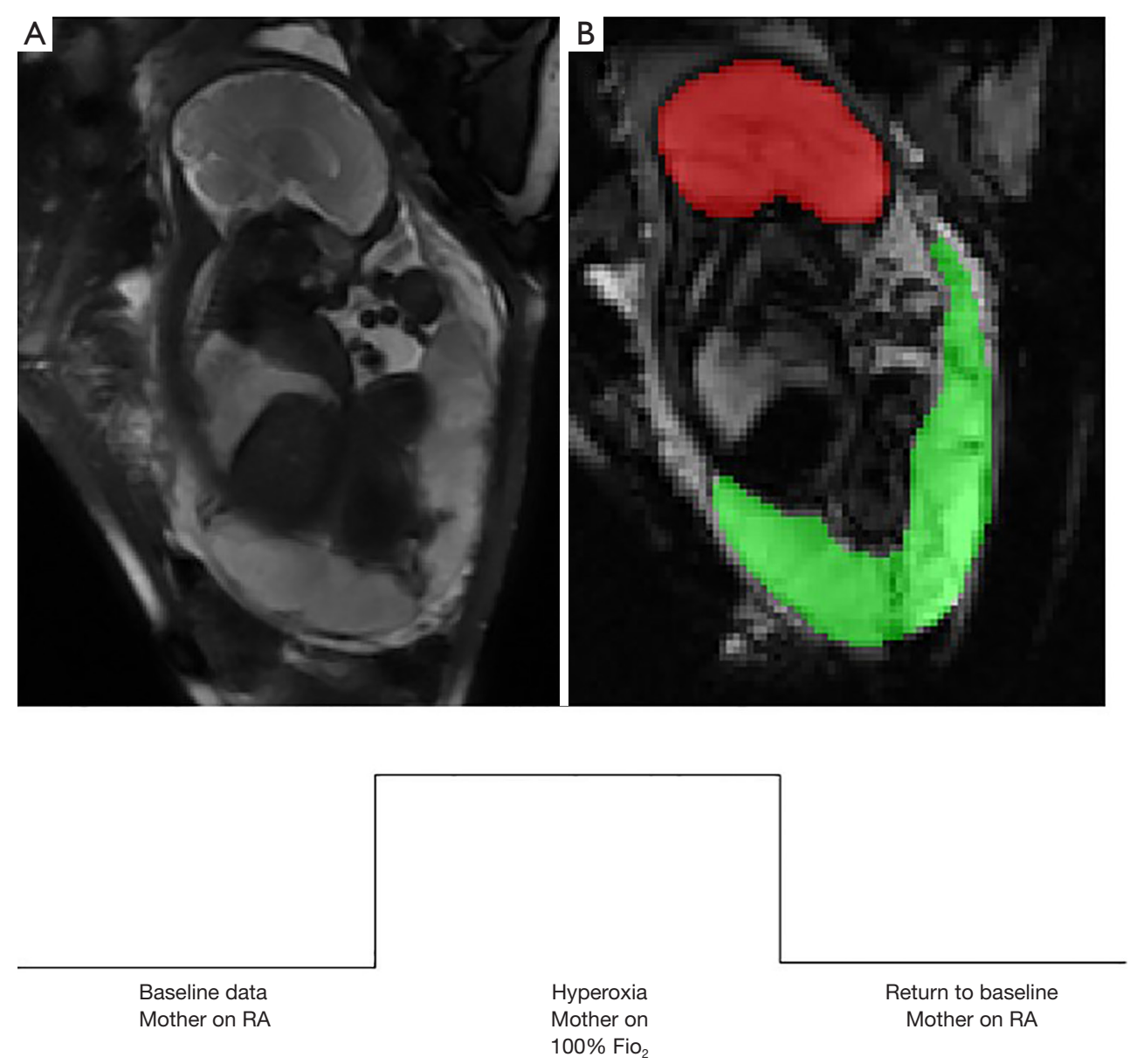

Figure 5 Anatomic image of fetus and placenta (A) and blood oxygen level dependent (BOLD) image of fetus and placenta (B) at 36 weeks gestation. Panel B highlights the fetal brain (red) and placenta (green) as regions of interest to measure BOLD signal. The maternal hyperoxia design collects BOLD signal at baseline, during maternal hyperoxia, and during return to baseline. Differences in BOLD signal reflect differences in regional oxygenation during each phase, which can be used to assess both baseline oxygenation, as well as capacity for oxygen transfer from mother to placenta and then fetus.

onset and progression of placental dysfunction in CHD is necessary, as is a better understanding of the direct impact of placental health on fetal well-being. As new research emerges with the intent to augment placental function in high-risk pregnancies, the biologic mechanisms underlying placental abnormalities in CHD will guide future clinicians on the relevance and applicability of novel placental treatments to this population. Moreover, successful development of early biomarkers of placental failure in the fetus with CHD is needed and will demand a comprehensive understanding of normal in vivo placental function across gestation, from which to reliably identify the onset and progression of placental dysfunction. Given the multi-factorial nature of morbidity related to $\mathrm{CHD}$, the future will require a multi-factorial approach to both care and prevention.

\section{Acknowledgments}

We would like to thank Ben Scalise for his talented artwork. Funding: None.

\section{Footnote}

Provenance and Peer Review: This article was commissioned by the Guest Editor (Antonio F. Corno) for the series "Prenatal Diagnosis in Congenital Heart Defects" published in Translational Pediatrics. The article has undergone external 
peer review.

Conflicts of Interest: Both authors have completed the ICMJE uniform disclosure form (available at http://dx.doi. org/10.21037/tp-20-347). The series "Pre-natal Diagnosis in Congenital Heart Defects" was commissioned by the editorial office without any funding or sponsorship. The authors have no other conflicts of interest to declare.

Ethical Statement: The authors are accountable for all aspects of the work in ensuring that questions related to the accuracy or integrity of any part of the work are appropriately investigated and resolved.

Open Access Statement: This is an Open Access article distributed in accordance with the Creative Commons Attribution-NonCommercial-NoDerivs 4.0 International License (CC BY-NC-ND 4.0), which permits the noncommercial replication and distribution of the article with the strict proviso that no changes or edits are made and the original work is properly cited (including links to both the formal publication through the relevant DOI and the license). See: https://creativecommons.org/licenses/by-nc-nd/4.0/.

\section{References}

1. Hahn D, Blaschitz A, Korgun ET, et al. From maternal glucose to fetal glycogen: expression of key regulators in the human placenta. Mol Hum Reprod 2001;7:1173-8.

2. Herrera E, Amusquivar E, Lopez-Soldado I, et al. Maternal lipid metabolism and placental lipid transfer. Horm Res 2006;65 Suppl 3:59-64.

3. Settle P, Mynett K, Speake P, et al. Polarized lactate transporter activity and expression in the syncytiotrophoblast of the term human placenta. Placenta 2004;25:496-504.

4. Randhawa R, Cohen P. The role of the insulin-like growth factor system in prenatal growth. Mol Genet Metab 2005;86:84-90.

5. Clifton VL, Read MA, Leitch IM, et al. Corticotropinreleasing hormone-induced vasodilatation in the human fetal placental circulation. J Clin Endocrinol Metab 1994;79:666-9.

6. Toriola AT, Vaarasmaki M, Lehtinen M, et al. Determinants of maternal sex steroids during the first half of pregnancy. Obstet Gynecol 2011;118:1029-36.

7. Bronson SL, Bale TL. The Placenta as a Mediator of Stress Effects on Neurodevelopmental Reprogramming.
Neuropsychopharmacology 2016;41:207-18.

8. Heerema-McKenney A. Defense and infection of the human placenta. APMIS 2018;126:570-88.

9. Thornburg KL, Kolahi K, Pierce M, et al. Biological features of placental programming. Placenta 2016;48 Suppl 1:S47-S53.

10. Thornburg KL, Marshall N. The placenta is the center of the chronic disease universe. Am J Obstet Gynecol 2015;213:S14-20.

11. Linask KK. The heart-placenta axis in the first month of pregnancy: induction and prevention of cardiovascular birth defects. J Pregnancy 2013;2013:320413.

12. Burton GJ, Jauniaux E. Development of the Human Placenta and Fetal Heart: Synergic or Independent? Front Physiol 2018;9:373.

13. Matthiesen NB, Henriksen TB, Agergaard P, et al. Congenital Heart Defects and Indices of Placental and Fetal Growth in a Nationwide Study of 924422 Liveborn Infants. Circulation 2016;134:1546-56.

14. Rychik J, Goff D, McKay E, et al. Characterization of the Placenta in the Newborn with Congenital Heart Disease: Distinctions Based on Type of Cardiac Malformation. Pediatr Cardiol 2018;39:1165-71.

15. Jones HN, Olbrych SK, Smith KL, et al. Hypoplastic left heart syndrome is associated with structural and vascular placental abnormalities and leptin dysregulation. Placenta 2015;36:1078-86.

16. Salafia CM, Zhang J, Charles AK, et al. Placental characteristics and birthweight. Paediatr Perinat Epidemiol 2008;22:229-39.

17. Madden JV, Flatley CJ, Kumar S. Term small-forgestational-age infants from low-risk women are at significantly greater risk of adverse neonatal outcomes. Am J Obstet Gynecol 2018;218:525.e1-e9.

18. Yu J, Flatley C, Greer RM, et al. Birth-weight centiles and the risk of serious adverse neonatal outcomes at term. J Perinat Med 2018;46:1048-56.

19. Tsai LY, Chen YL, Tsou KI, et al. The impact of smallfor-gestational-age on neonatal outcome among very-lowbirth-weight infants. Pediatr Neonatol 2015;56:101-7.

20. Albalawi A, Brancusi F, Askin F, et al. Placental Characteristics of Fetuses With Congenital Heart Disease. J Ultrasound Med 2017;36:965-72.

21. Hayward CE, Lean S, Sibley CP, et al. Placental adaptation: what can we learn from birthweight:placental weight ratio? Front Physiol 2016;7:28.

22. Ismail KI, Hannigan A, O'Donoghue K, et al. Abnormal placental cord insertion and adverse pregnancy outcomes: a 
systematic review and meta-analysis. Syst Rev 2017;6:242.

23. Buchanan-Hughes A, Bobrowska A, Visintin C, et al. Velamentous cord insertion: results from a rapid review of incidence, risk factors, adverse outcomes and screening. Syst Rev 2020;9:147.

24. Ravikumar G, Crasta J, Prabhu JS, et al. Eccentric placentae have reduced surface area and are associated with lower birth weight in babies small for gestational age. J Dev Orig Health Dis 2018;9:281-6.

25. Luo G, Redline RW. Peripheral insertion of umbilical cord. Pediatr Dev Pathol 2013;16:399-404.

26. Miremberg H, Gindes L, Schreiber L, et al. The association between severe fetal congenital heart defects and placental vascular malperfusion lesions. Prenat Diagn 2019;39:962-7.

27. Redline RW, Ravishankar S. Fetal vascular malperfusion, an update. APMIS 2018;126:561-9.

28. Redline RW, Pappin A. Fetal thrombotic vasculopathy: the clinical significance of extensive avascular villi. Hum Pathol 1995;26:80-5.

29. Redline RW. Distal villous immaturity. Diagnostic Histopathology 2012;18:189-94.

30. Ruiz A, Ferrer Q, Sanchez O, et al. Placenta-related complications in women carrying a foetus with congenital heart disease. J Matern Fetal Neonatal Med 2016;29:3271-5.

31. DeVore GR. The importance of the cerebroplacental ratio in the evaluation of fetal well-being in SGA and AGA fetuses. Am J Obstet Gynecol 2015;213:5-15.

32. Baschat AA, Gembruch U. The cerebroplacental Doppler ratio revisited. Ultrasound Obstet Gynecol 2003;21:124-7.

33. Morales-Roselló J, Khalil A, Morlando M, et al. Changes in fetal Doppler indices as a marker of failure to reach growth potential at term. Ultrasound Obstet Gynecol 2014;43:303-10.

34. Odibo AO, Riddick C, Pare E, et al. Cerebroplacental Doppler ratio and adverse perinatal outcomes in

Cite this article as: Andescavage NN, Limperopoulos C. Placental abnormalities in congenital heart disease. Transl Pediatr 2021;10(8):2148-2156. doi: 10.21037/tp-20-347 intrauterine growth restriction: evaluating the impact of using gestational age-specific reference values. J Ultrasound Med 2005;24:1223-8.

35. Donofrio MT, Bremer YA, Schieken RM, et al. Autoregulation of cerebral blood flow in fetuses with congenital heart disease: the brain sparing effect. Pediatr Cardiol 2003;24:436-43.

36. Mebius MJ, Clur SAB, Vink AS, et al. Growth patterns and cerebroplacental hemodynamics in fetuses with congenital heart disease. Ultrasound Obstet Gynecol 2019;53:769-78.

37. Andescavage N, Yarish A, Donofrio M, et al. 3-D volumetric MRI evaluation of the placenta in fetuses with complex congenital heart disease. Placenta 2015;36:1024-30.

38. Andescavage $\mathrm{N}$, duPlessis A, Metzler M, et al. In vivo assessment of placental and brain volumes in growthrestricted fetuses with and without fetal Doppler changes using quantitative 3D MRI. J Perinatol 2017;37:1278-84.

39. Zun Z, Zaharchuk G, Andescavage NN, et al. Noninvasive placental perfusion imaging in pregnancies complicated by fetal heart disease using velocity-selective arterial spin labeled MRI. Sci Rep 2017;7:16126.

40. Figueras F, Caradeux J, Crispi F, et al. Diagnosis and surveillance of late-onset fetal growth restriction. Am J Obstet Gynecol 2018;218:S790-S802.e1.

41. Sun L, Macgowan CK, Sled JG, et al. Reduced fetal cerebral oxygen consumption is associated with smaller brain size in fetuses with congenital heart disease. Circulation 2015;131:1313-23.

42. You W, Andescavage NN, Kapse K, et al. Hemodynamic responses of the placenta and brain to maternal hyperoxia in fetuses with congenital heart disease by using blood oxygen-level dependent MRI. Radiology 2020;294:141-8.

43. Heazell AE, Whitworth M, Duley L, et al. Use of biochemical tests of placental function for improving pregnancy outcome. Cochrane Database Syst Rev 2015;(11):CD011202. 